\title{
POLYNOMIAL ROOT-FINDING ALGORITHMS AND BRANCHED COVERS田
}

\author{
MYONG-HI KIM团AND SCOTT SUTHERLAND目
}

\begin{abstract}
We construct a family of root-finding algorithms which combine knowledge of the branched covering structure of a polynomial with a path-lifting algorithm for finding individual roots. In particular, the family includes an algorithm that computes an $\epsilon$-factorization of a polynomial of degree $d$ which has an arithmetic complexity of $\mathcal{O}\left(d(\log d)^{2}|\log \epsilon|+d^{2}(\log d)^{2}\right)$. At the present time, this complexity is the best known in terms of the degree.
\end{abstract}

Key words. Newton's method, approximate zeros, arithmetic complexity, path-lifting method, branched covering.

AMS subject classifications. 68Q25; Secondary 58C10, 65H05, 30C15, 58F08

Introduction. The problem of devising optimal methods for numerically approximating the roots of a polynomial has been of interest for several centuries, and is far from solved. There are numerous recent works on root-finding algorithms and their cost, for example, the work of Jenkins and Traub [एT70], Renegar [Ren87], Schönhage [Sch82], and Shub and Smale [SS85, 5S86, Sma85. This list is far from complete; the reader should refer to the aforementioned papers as well as [DH69] for more detailed background. The work in this paper is most closely related to that of Smale.

Our algorithm computes an approximate factorization of a given polynomial (that is, it approximates all the roots). In constructing it, we combine global topological information about polynomials (namely, that they act as branched covers of the Riemann Sphere) with a path-lifting method for finding individual roots. Utilizing this global information enables us to use fewer operations than applying the path-lifting method to each root sequentially.

Renegar's algorithm in Ren87 approximates all $d$ roots of a given polynomial using $\mathcal{O}\left(d^{3} \log d+d^{2}(\log d)(\log |\log \epsilon|)\right)$ arithmetic operations in the worst case. He has shown that the factor of $\log |\log \epsilon|$ in the complexity is the best possible if one restricts to the operations,,$+- \times$, and $\div$. This algorithm has a component (the Shur-Cohn algorithm) which requires exact computation and so is not suitable for an analysis of bit complexity, that is, one which accounts for rounding errors introduced by finite precision. In Pan87, Pan gives a different algorithm which slightly improves the complexity to $\mathcal{O}\left(d^{2} \log d \log |\log \epsilon|\right)$; this algorithm also operates effectively as a parallel algorithm.

Schönhage Sch82 gives an algorithm which produces an $\epsilon$-factorization with a

${ }^{*}$ Received by the editors July 12, 1991.

$\dagger$ An earlier version of this paper was circulated with the title "Parallel Families of Polynomial Root-Finding Algorithms".

¥ Institute For Mathematical Sciences, SUNY Stony Brook, Stony Brook, NY 11794-3660. email: myonghi@math. sunysb. edu and scott@math. sunysb.edu.

$\S$ Part of this work was done while M. Kim was at Bellcore, Morristown, NJ. 
bit complexity of $\mathcal{O}\left(d^{3} \log d+d^{2}|\log \epsilon|\right) \log (d|\log \epsilon|) \log \log (d|\log \epsilon|)$, via the "splitting circle method". Note that the customary parameter for bit length of the coefficients does not appear in the complexity. This is because, as Schönhage states, for fixed degree $d$ and output precision $\epsilon$, there is a number $s_{0}$ for which "the input [coefficients] $a_{\nu}$ can be restricted to complex integer multiples of $2^{-s_{0}}$ without loss of generality." In [Ren87, it is stated that Schönhage believes that, if exact arithmetic is used, this method "should yield a complexity bound [in $\epsilon]$ of $\mathcal{O}\left(d^{\alpha} \log |\log \epsilon|\right)$, most likely with $\alpha \leq 3$."

Smale's path lifting algorithm in [Sma85] approximates a single root of the polynomial with a worst case arithmetic complexity of $\mathcal{O}\left(d(\log d)|\log \epsilon|+d^{2}(\log d)^{2}\right)$, and an average complexity of $\mathcal{O}\left(d^{2}+d|\log \epsilon|\right)$. One good feature of this line of work is that it is stable under round-off error. In Kim89a, Kim shows that if $f$ and $f^{\prime}$ are computed with relative error $10^{-3}$ until an approximate zero (see Section 1.2) is reached, then the algorithm behaves exactly the same. A recent series of papers by Shub and Smale SS93a, SS93b, SS93c, SS93d] generalizes the path lifting algorithm to systems of homogeneous polynomials in several variables.

The algorithm presented here exploits the branched covering structure of a polynomial to choose good starting points for a variant of Smale's algorithm, and we obtain a worst case arithmetic complexity of $\mathcal{O}\left(d(\log d)|\log \epsilon|+d^{2}(\log d)^{2}\right)$ to compute an $\epsilon$-factorization. In a subsequent paper, we shall compute the bit complexity of this algorithm. Because of the stability mentioned in the previous paragraph and the ability to exploit bounds on the variation of $f$ and $f^{\prime}$, we hope to achieve results comparable to Schönhage's.

At first glance, it may appear that our complexity results are inferior to some of those above in terms of $\epsilon$. However, in practice there is usually a relationship between the degree $d$ and the desired precision $\epsilon$; if we have $\epsilon \geq 2^{-d}$, then the complexity of our algorithm compares favorably with all of those mentioned above. Furthermore, our algorithm is quite simple to implement and is numerically very stable.

Our algorithm is suitable for some amount of parallelization, but has a sequential component of $\mathcal{O}(d+|\log \epsilon|)$ operations. However, we think of this algorithm as acting on $d$ points simultaneously, and techniques which evaluate a polynomial at $d$ points (see BM75, for example) are used to cut the cost involved. Of course, the algorithm can be implemented on a sequential machine while still taking advantage of these techniques. In fact, evaluation of the polynomial is the only point at which we at which we need to use asymptotic estimates to achieve the stated complexity; the other places where we use asymptotic estimates are only for ease of expositon.

The reader should also see the papers BFKT88, BT90, Nef90 for fully parallel algorithms for solving polynomials with integer coefficients. In [BFKT88, it is shown that if all roots of the polynomial are real, this problem is in NC. Neff extends this result to allow complex roots in Nef90.

This paper is structured as follows: In Chapter 1, after some background material, we recall the "path lifting method" of Smale and present a version of the relevant 
theorem (our Theorem 1.5) which improves the constants involved somewhat. We then discuss how we can exploit the branched-covering structure of a polynomial to choose initial points for the algorithm, many of which will converge to roots. We close the chapter with a brief explanation of how to construct families of algorithms which locate $d / n$ roots at a time, for various values of $n$.

Chapter 2 presents an explicit algorithm for a specific family, which locates $d / 2$ points at a time. Our main theorem, Theorem 2.1, states that this algorithm always terminates with an $\epsilon$-factorization of the input polynomial, and gives a bound on the number of arithmetic operations required in the worst case. As a corollary, the algorithm can be used to locate all $d$ roots of the polynomial to within $\epsilon$ with a complexity of $\mathcal{O}\left(d^{2}(\log d)|\log \epsilon|+d^{2}(\log d)^{2}\right)$. In the subsequent sections, each component of the algorithm is analyzed, and the relevant lemmas are proven. Finally, we tie all the components together and prove the main theorem.

\section{Preliminaries.}

1.1. Root and coefficient bounds. Given a polynomial $\phi(z)=\sum_{i=0}^{d} a_{i} z^{i}$, with $a_{i} \in \mathbb{C}$, it is our goal to determine an approximate factorization of $\phi$, that is, approximations $\hat{\xi}_{i}$ to the actual roots $\xi_{i}$ of $\phi$ so that $\left\|\phi(z)-\Pi\left(z-\hat{\xi}_{i}\right)\right\|<\epsilon$. The norm we shall use here is the max-norm, that is, $\|\phi\|=\max \left|a_{j}\right|$. A related problem is to ensure that $\left|\xi_{i}-\hat{\xi}_{i}\right|<\epsilon^{\prime}$; there are well-known estimates giving the relationship between $\epsilon$ and $\epsilon^{\prime}$, so solving one problem essentially solves the other.

In order to have an estimate on the complexity of a root-finding algorithm, we need a compactness condition on the space of polynomials. This can be done either by placing conditions of the location of the roots or on the coefficients; such bounds are interrelated.

Since our goal is to minimize a functional norm, it seems most natural to place our conditions on the coefficients. We shall assume our input polynomial $\phi$ is an element of the family

$$
\mathcal{P}_{d}(1)=\left\{z^{d}+\sum_{j=0}^{d-1} a_{j} z^{j}, \text { with }\left|a_{j}\right| \leq 1\right\} .
$$

This is the same space as used by Smale and others [SS85, Sma81, Sma85]. One can always transform an arbitrary polynomial into an element of $\mathcal{P}_{d}(1)$ : if $p(z)=\sum_{i=0}^{d} b_{i} z^{i}$, and $B=\max \left|b_{j} / b_{d}\right|^{1 /(d-j)}$, then $p(B z) / B^{d} \in \mathcal{P}_{d}(1)$.

One should not confuse this family with the degree $d$ polynomials whose roots are in the unit disk, although unfortunately this space is also often denoted by $\mathcal{P}_{d}(1)$ (for example, in [Fri90 and [Ren87).

There are a number of estimates which relate the coefficients of a polynomial to a bound on the modulus of the zeros (see [Hen74] or Mar66, for example). Such an estimate is important to us, since although membership in $\mathcal{P}_{d}(1)$ is not preserved under deflation (division of factors), bounds on the modulus of the roots are. We state one such bound here (Corollary $6.4 \mathrm{k}$ of $\operatorname{Hen} 74$ ): 
LEMMA 1.1. All the zeros of the polynomial $z^{d}+\sum_{j=0}^{d-1} a_{j} z^{j}$ lie within the open disk with center 0 and radius

$$
2 \max _{0 \leq j<d}\left|a_{j}\right|^{1 /(d-j)} .
$$

As an immediate consequence, we see that the roots of a polynomial in $\mathcal{P}_{d}(1)$ lie within $\mathbb{D}_{2}$.

1.2. Approximate zeros. Our algorithm uses a path lifting method (see below) to get close to the roots of our polynomial, and then uses the standard Newton's method to further refine these approximations. This is done because Newton's method converges very quickly in a neighborhood of a simple root, but can fail for some initial points outside this neighborhood. One of the authors Sut89 has shown how one can guarantee convergence of Newton's method, but a bound on the arithmetic complexity has not been computed. Instead, we use the more certain path lifting method as described in Section 1.3; this allows an explicit computation of the complexity.

Following Smale [Sma81], we call a point $z_{0}$ an approximate zero if Newton's method converges rapidly (that is, quadratically) when started from $z_{0}$. Such terminology is reasonable, because given such a point, we can quickly obtain an approximation of a root to arbitrary precision.

Definition 1.2. Let $f$ be a polynomial and let $z_{n}$ be the $n^{\text {th }}$ iterate under Newton's method of the point $z_{0}$, that is, $z_{n}=z_{n-1}-f\left(z_{n-1}\right) / f^{\prime}\left(z_{n-1}\right)$. Then we say that $z_{0}$ is an approximate zero of $f$ if, for all $n>0$ we have

$$
\left|z_{n}-\zeta\right| \leq 8\left(\frac{1}{2}\right)^{2^{n}}\left|z_{0}-\zeta\right|
$$

for some root $\zeta$ of $f$.

Notice that this definition is never satisfied in the neighborhood of a multiple root of $f$, since the convergence of Newton's method is asymptotically linear there. In our algorithm, we perturb the polynomial slightly to ensure that we always have simple zeros. Refer to Section 2.1 and Section 2.3 for more details.

Kim [Kim88] and Smale [Sma86] have developed readily tested criteria for determining, based on the values of the derivatives $f^{(k)}(z)$, when a point $z$ is an approximate zero. These can be extended to a much more general setting, namely for $f$ a mapping between Banach spaces. The following is essentially Theorem A of [Sma86]:

LEMMA 1.3. Let

$$
\alpha_{f}(z)=\max _{k>1}\left|\frac{f(z)}{f^{\prime}(z)}\right|\left|\frac{f^{(k)}(z)}{k ! f^{\prime}(z)}\right|^{1 /(k-1)} .
$$

If $\alpha_{f}(z)<\frac{1}{8}$, then $z$ is an approximate zero of $f$.

We will find the following also very useful. 
LEMMA 1.4. Let $f(z)$ be a polynomial and $z$ be a complex number so that $f^{\prime}(z) \neq 0$, and let $R_{f}(z)$ be the radius of convergence of the branch of the inverse $f_{z}^{-1}$ which takes $f(z)$ to $z$. If

$$
\frac{|f(z)|}{R_{f}(z)}<\frac{1}{10}
$$

then $z$ is an approximate zero of $f$. Furthermore, if we have $|f(z)| / R_{f}(z)<1 / 32$, then $\alpha_{f}(z)<1 / 8$.

REMARK. If Smale's mean value conjecture holds (see [Sma81, Tis89]), then the hypotheses of the lemma imply that $\alpha_{f}(z)<1 / 8$.

Proof. The first result is a consequence of the proof of Theorem 4.4 of Kim88, and the second is an immediate consequence of Corollary 4.3 of the same paper. This, in turn, uses the Extended Loewner's Theorem in [Sma81].

1.3. The path lifting method. Here we review the path lifting method, which forms the core of our iteration scheme. This method is sometimes referred to as a "generalized Euler method" or "modified Newton's method"; we prefer the term "path lifting method" as it is the most descriptive (to us, anyway). This method appears in the work of Steven Smale [Sma85], although the version we present here is slightly different and we present another proof of the relevant theorem, which is quite simple. It should be emphasized that the path lifting method, like Newton iteration, is an algorithm for finding a single root of a polynomial; we discuss how to combine these to find all roots in Section 1.5 below.

We think of a polynomial $f$ as a map from the source space to the target space; that is, $f: \mathbb{C}_{\text {source }} \rightarrow \mathbb{C}_{\text {target }}$. Given an initial value $z_{0}$ in the source space, we connect its image $w_{0}=f\left(z_{0}\right)$ to 0 in the target space, and then lift this ray under the proper branch of $f^{-1}$ to a path connecting $z_{0}$ with a root $\zeta$ of $f$. Of course, we don't explicitly know this inverse, but if the path in the target space stays well away from the critical values of $f$, the local inverse map $f_{z_{0}}^{-1}$ is well-defined on a neighborhood of the ray. Even if the path does contain critical values, a local inverse can still be defined for some $z_{0}$. See Section 1.4.

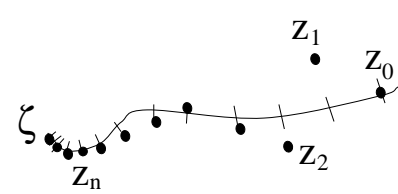

source space

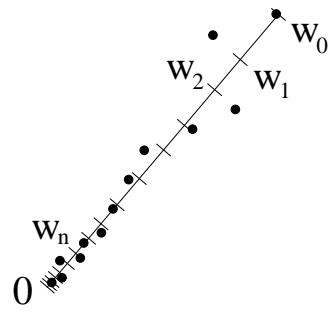

target space

FIG. 1.1. The source and target spaces in the path lifting method. In the source space, each $z_{i}$ is indicated by a black dot, and $f\left(z_{i}\right)$ is indicated by a black dot in the target space. Similarly, the $w_{i}$ are indicated by tick marks in the target space, and $f^{-1}\left(w_{i}\right)$ by ticks in the source space. 
The basic idea of the path lifting method is to take a sequence of points $w_{n}$ along the ray in the target space, with $w_{0}=f\left(z_{0}\right)$. We then construct a sequence of points $z_{n}$ in the source space so that $f\left(z_{n}\right)$ is near $w_{n}$ in the target. This is done using a single step of Newton's method to solve $f(z)=w_{n}$ with $z_{n-1}$ as the starting point. That is,

$$
z_{n}=z_{n-1}-\frac{f\left(z_{n-1}\right)-w_{n-1}}{f^{\prime}\left(z_{n-1}\right)} .
$$

This construction will converge to a root $\zeta$ in the source space if there is a wedge about the ray in the target space on which there is a well-defined branch of the inverse $f_{\zeta}^{-1}$, and if the $w_{n}$ are chosen properly (in a way which depends on the angle of the wedge). The larger the wedge about the ray, the faster the method converges. We now state the exact theorem, although we shall defer the proof until Section 2.3.

Notation. By a wedge $\mathcal{W}_{A, w}$, we mean the set

$$
\{z|0<| z|<2| w \mid, \arg w-A<\arg z<\arg w+A\} .
$$

THEOREM 1.5. Suppose that the branch of the inverse $f_{z_{0}}^{-1}$ is analytic on a wedge $\mathcal{W}_{A, w_{0}}$, with $0<A \leq \pi / 2$, and let $h \leq \frac{\sin A}{19}$. Suppose also that $\left|f\left(z_{0}\right)-w_{0}\right|<h\left|w_{0}\right| / 2$, and define

$$
w_{n}=(1-h)^{n} w_{0}, \quad z_{n+1}=z_{n}-\frac{f\left(z_{n}\right)-w_{n+1}}{f^{\prime}\left(z_{n}\right)} .
$$

Then $\left|f\left(z_{n}\right)-w_{n}\right| \leq h\left|w_{n}\right| / 2$ and $z_{n+1} \in f_{z_{0}}^{-1}\left(\mathcal{W}_{A, w_{n}}\right)$.

It should be noted that this theorem is a slight improvement of Smale's Theorem A in Sma85. His proof is valid for all angles, but is stated only for $A=\pi / 12$ with $h=1 / 98$, and for $A=\pi / 4$ with $h=1 / 32$. For $A=\pi / 12$ we can take take $h=1 / 74$, and for $A=\pi / 4, h=1 / 27$ is adequate.

1.4. Branched covers, inverse functions, and all that. If $f$ is a polynomial of degree $d$, then $f: \mathbb{C} \rightarrow \mathbb{C}$ is a branched covering with branch points at the critical points $\theta_{i}$ of $f$. If $z$ is a regular point of $f$, that is, $f^{\prime}(z) \neq 0$, then there is a well-defined inverse function $f_{z}^{-1}$ so that $f_{z}^{-1}(f(z))=z$.

In any neighborhood of a critical point of $f$, there cannot be a single valued inverse; however, the behavior at such points is well understood. Let $\theta$ be a critical point of multiplicity $k-1$. Then we have

$$
f(z)-f(\theta)=(z-\theta)^{k} g(z), \quad \text { where } \quad g(\theta) \neq 0 .
$$

One can then define $k$ branches of the inverse which are analytic on a small slit disk about $f(\theta)$. We may, of course, choose any slit which connects $f(\theta)$ to the boundary of the disk. The reader is referred to a complex analysis text for further details (for example, see Ahl79). 
Let $\left\{\zeta_{j}\right\}$ be the $d$ roots of $f$, represented with multiplicity. If $\zeta_{j}$ is a simple root, denote by $f_{\zeta_{j}}^{-1}$ or $f_{j}^{-1}$ the unique branch of the inverse of $f$ which takes 0 to $\zeta_{j}$. On the other hand, if $\zeta_{j}$ is a multiple root, we let $f_{\zeta_{j}}^{-1}=f_{j}^{-1}$ be one of the branches of the inverse that take 0 to $\zeta_{j}$, taking care to account for all such branches exactly once. This can be done, since if $\zeta$ is a root of multiplicity $k \geq 2$, it is also a critical point of multiplicity $k-1$, and so there are $k$ branches of the inverse.

We now analytically continue each of the $f_{j}^{-1}$ to a maximal starlike domain $\Omega_{j}$ in the target space; that is, we attempt to extend each $f_{j}^{-1}$ along open rays from 0 . When doing this, it is useful to think of the target space as consisting of $d$ copies of $\mathbb{C}$, with a single $f_{j}^{-1}$ associated to each one. When does the analytic continuation fail? Precisely when the inverse image of a ray encounters a critical point of $f$. Refer to Figure 1.2.

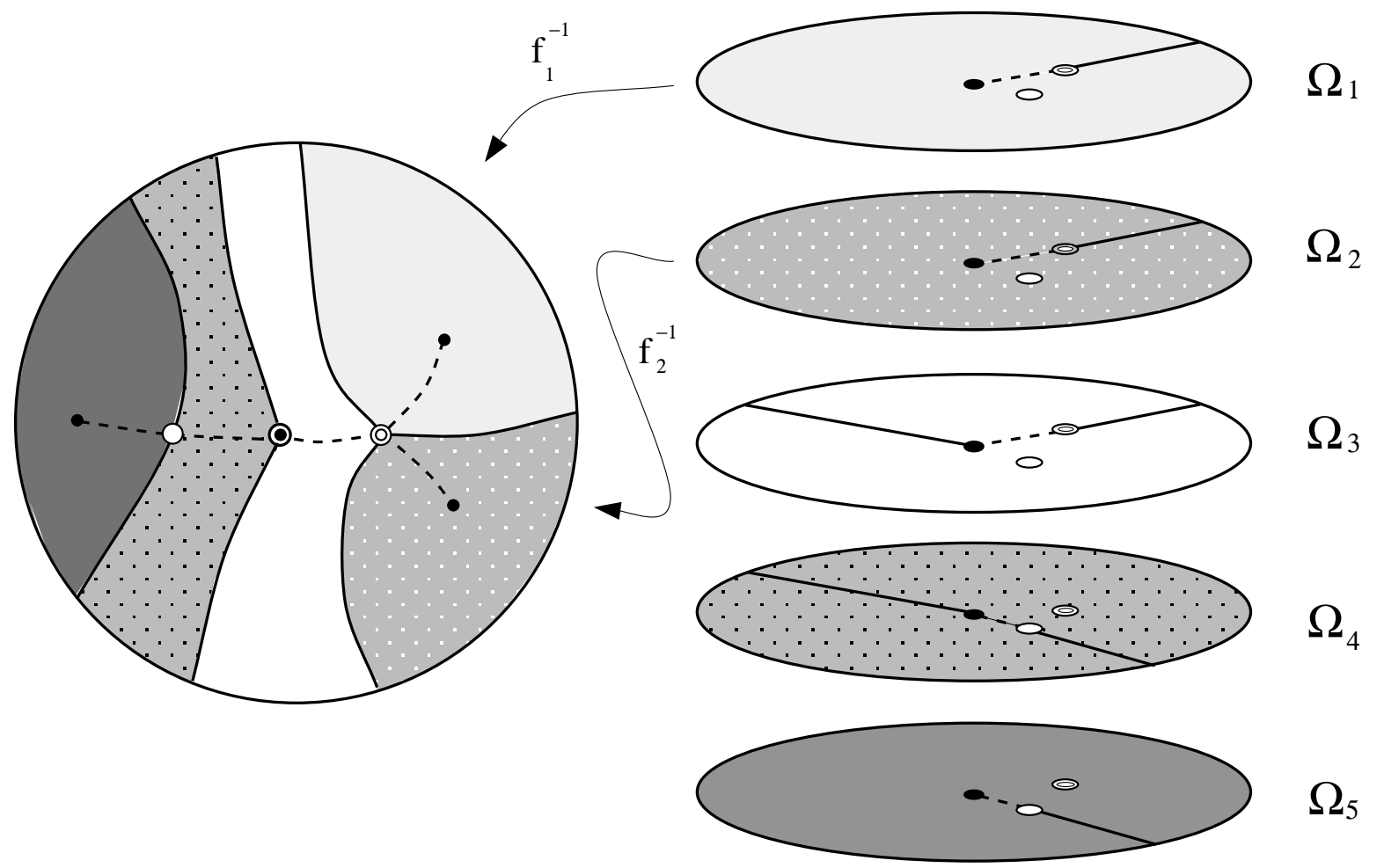

FIG. 1.2. The $\Omega_{j}$ in the target space (on the right), and the corresponding source space, for a degree 5 polynomial with a double root (black dot inside a white dot) and a critical point of multiplicity 2 (double white dot). The other critical point is marked by a single white dot, and the roots by black dots. The cuts in the $\Omega_{j}$ are represented by black radial lines.

At this point, it may be useful to consider the Newton vector field given by

$$
\dot{z}=-f(z) / f^{\prime}(z) \text {. }
$$

Let $\varphi_{t}(z)$ be the solution curve with initial condition $\varphi_{0}(z)=z$. Then we have

$$
f\left(\varphi_{t}(z)\right)=\mathrm{e}^{-t} f(z)
$$

that is, $f$ maps solution curves of the Newton vector field to rays in the target space. Notice that the singularities of the vector field occur precisely at the points where 
$f^{\prime}(z)=0$. The solution curves $\varphi_{t}(z)$ which have singularities play an important role here: they divide the source space into regions on which $f$ is injective. Refer to Shu83, STW88, Sma85 for more details on the behavior of the solution curves. Applying the path lifting method can be viewed as attempting to follow the solution curves to the flow $\varphi_{t}$.

When constructing the $\Omega_{j}$, we continue $f_{j}^{-1}$ radially outward until a critical point $\theta$ is encountered in the source space. We then exclude the ray $\{r f(\theta) \mid r \geq 1\}$ from $\Omega_{j}$, and continue by moving along rays which avoid the cut. Notice that when we encounter a critical point $\theta$ of multiplicity $k-1$, we need to slit at most $k$ of the $\Omega_{j}$ starting at $f(\theta)$. Also, note that some of the $\Omega_{j}$ may already be slit at $f(\theta)$, since there may be another critical point whose image lies on the same ray.

We now count the number of such cuts: $f$ has $d-1$ critical points (with multiplicity), and a critical point of multiplicity $k-1$ can cause at most $k$ cuts. This means we have at most $2(d-1)$ cuts, distributed through the $d$ copies of the target space. Note that if $\Omega_{j}$ contains some wedge $W$, then $f_{j}^{-1}$ is analytic on $W$. The following counts the number of $\Omega_{j}$ which contain wedges of a given size.

Lemma 1.6. Let $m$ be an integer, and divide $\mathbb{C}$ into $m$ wedges

$$
W_{n, m}=\left\{w \mid \frac{2 n \pi}{m}<\arg w<\frac{2(n+1) \pi}{m}\right\} \quad n=0, \ldots, m-1 .
$$

For each wedge $W_{n, m}$, let $N(n, m)$ be the number of $\Omega_{j}$ which contain the sector $W_{n, m}$, and let $N(m)=\max _{0 \leq n<m} N(n, m)$. Then

$$
N(m) \geq d-\left\lfloor\frac{2(d-1)}{m}\right\rfloor
$$

Proof. Since we have $2(d-1)$ cuts and $m$ wedges $W_{n}$, at least one of the wedges has no more than $\frac{2(d-1)}{m}$ cuts. Since there are $d \Omega_{j}$ s, we have the result.

Corollary 1.7. $N(d)=d, N(3) \geq \frac{d}{3}$, and $N(4) \geq \frac{d}{2}$.

Proof. Application of the formula above gives the result for $N(3)$ and $N(4)$, and yields $N(d) \geq d-1$. However, since each critical point causes at least two cuts, it is not possible to have a wedge cut only once. Thus, the value $d-1$ is not permissible for $N(d)$, giving $N(d)=d$.

1.5. Families of root-finding methods. We now use the result of Lemma 1.6 to construct families of root-finding algorithms. Recall that the the modified Newton method described in Section 1.3 works when there is an $\Omega_{j}$ containing a wedge about our initial value $f\left(z_{0}\right)$; the larger the wedge, the faster the method converges.

For each family, we start with $m d$ points in the source space placed around a circle which contains all the roots. We think of this as $m$ sets of $d$ initial points, and choose them so that the image of each set lies well inside each of the $m$ sectors $W_{n, m}$ in the target space. Then by Lemma 1.6, one of the $m$ sets of points will contain at least 
$N(m)$ elements whose images are each in a "good wedge", that is, they lie in some $\Omega_{j}$. As a consequence, iterating these points under the path lifting method will locate at least $N(m)$ roots of the polynomial.

Particular families of interest are $m=d$, which gives the algorithm discussed in Kim89b, and $m=4$, on which we focus our attention here. The basic idea of all of the algorithms is this: obtain md "good" initial points and apply the path lifting method to $d$ of them at a time. If, after a prescribed number of iterations, we have found approximation to at least $N(m)$ roots (counting multiplicity), we deflate the polynomial (that is, divide out the approximated roots) and repeat the process on the result. If not, we try again with the next set of $d$ points. Note that we are guaranteed success by the time we try the $m^{\text {th }}$ set.

The remainder of the paper consists of a detailed description and analysis of the algorithm for $m=4$. Most of what follows can be readily adapted to the other families as well.

\section{A root-finding algorithm.}

2.1. Statement of the algorithm and main theorem. Here we present our root-finding algorithm for the family $m=4$. The presentation is structured as a main routine and several subroutines, which do most of the work.

Notation. Throughout this chapter, we shall denote matrices, vectors, and sets in uppercase calligraphic type, and their elements in subscripted lowercase type. For example, $x_{j}$ is the $j^{\text {th }}$ element of the vector $\mathcal{X}$. We shall also use the notation $\lfloor x\rfloor$ to denote the least integer in $x$, sometimes also called floor $(x)$.

The main routine merely inputs the desired polynomial and precision, rescales it so the roots lie in the disk of radius $1 / 2$, then repeatedly calls a subroutine to halve the number of unknown roots (counted with multiplicity) and deflate. We do the rescaling in order to easily bound the error introduced by the FFT deflation. The set $\Lambda$ contains all the approximations found by the $i^{\text {th }}$ stage.

Note that the algorithm is given for an arbitrary monic polynomial, since only minor changes are required to normalize the input polynomial. If it is assumed that the input polynomial is already in $\mathcal{P}_{d}(1)$, we can take $f_{0}(z)=\phi(4 z) / 4^{d}$ and $\tau=32 \epsilon / 7^{d+3}$.

\section{Main Routine}

Input monic polynomial $\phi(z)=\sum_{i=0}^{d} a_{i} z^{i}$ and desired precision $\epsilon$.

Let $f_{0}(z)=\phi(K z) / K^{d}$, with $K=4 \max \left|a_{j}\right|^{\frac{1}{d-j}}$.

Let $\tau=\frac{\epsilon}{2 K^{d}}(4 / 7)^{d+3}$.

Let $i=0$ and $\Lambda=\emptyset$

While $\#(\Lambda)<\operatorname{deg}(\phi)$

Let $\left(f_{i+1}, \Lambda_{i}\right)=$ get-half-roots-and-deflate $\left(f_{i}, \tau\right)$.

Let $\Lambda=\Lambda \cup \Lambda_{i}$.

Increment $i$.

End While

Output $K \Lambda$. 
The function get-half-roots-and-deflate takes as input a normalized polynomial $f$ and precision $\tau$. It returns a set of points $y_{j}$ which approximate at least half of the roots of $f$ (with multiplicity) and a new polynomial $\tilde{f}$ which we obtain by deflation. These satisfy $\left\|f(z)-\tilde{f}(z) \prod\left(z-y_{j}\right)\right\|<2 \tau$. We should point out here that we are actually finding approximate zeros of $f-\vec{\tau}$, where $|\vec{\tau}|=\tau$, which depends on $\epsilon$. When the translation is in the proper quadrant, this will ensure that the relevant roots of $f-\vec{\tau}$ are simple, so that we have approximate zeros in a neighborhood. This allows us to obtain the right number of approximations to a multiple root, without worrying about winding number arguments or the like. We emphasize again that $\tau$ is chosen as a function of $\epsilon$, and is small enough that the approximation polynomial has negligible errors in the non-constant terms.

The matrix $\mathcal{Z}$ consists of 4 rows of $d$ "good" initial conditions, with $\left|z_{j, k}\right|=3 / 2$ and $\arg f\left(z_{j, k}\right) \approx 2 \pi \mathrm{i} j / 4$. We use $\mathcal{Z}_{j}$ to represent the $j^{\text {th }}$ row.

$$
\text { function get-half-roots-and-deflate }(f, \tau)
$$

Let $d=\operatorname{deg} f$.

Let $\mathcal{Z}=$ choose-4d-good-initial-points $(f)$.

For $j=1$ to 4 do

Let $\mathcal{Y}=$ iterate-PLM $\left(f, \mathcal{Z}_{j}, j, \tau\right)$.

Let $\psi=f-\tau \mathrm{e}^{2 \pi \mathrm{i} j / 4}$.

Let $\mathcal{X}=$ select-approx-zeros $(\psi, \mathcal{Y})$.

Let $\mathcal{W}=\operatorname{polish}-\operatorname{roots}(\psi, \mathcal{X}, \tau)$.

Let $\mathcal{V}=$ weed-out-duplicates $(\psi, \mathcal{W})$.

If $\#(\mathcal{V}) \geq d / 2$ then

Let $\tilde{f}=\operatorname{deflate}(\psi, \mathcal{V})$.

$\operatorname{Return}(\tilde{f}, \mathcal{V})$

End if

End for

Print "We have proven this statement will not be reached. Check your code."

Abort.

The function choose-4d-good-initial-points gives us our 4 sets of $d$ initial values on the circle of radius $3 / 2$. The sets have the property that elements of the same set are mapped to points with approximately the same argument, and elements of distinct sets are mapped to points in different quadrants of the target space. There are several different ways to accomplish this, and other methods can slightly decrease the number of operations required in iterate-PLM. Please refer to the remarks in Section 2.2 for more details.

\section{function choose-4d-good-initial-points $(f)$}

Let $N=676 \operatorname{deg} f$.

For $k=1$ to $N$

$$
\text { Let } \omega_{k}=\frac{3}{2} \mathrm{e}^{2 \pi \mathrm{i} k / N} \text {. }
$$

End For.

For $j=1$ to 4 
Let $\mathcal{Z}_{j}$ be the union of the $\omega_{k}$ for which $\arg f\left(\omega_{k}\right) \leq 2 \pi / j$ and $\arg f\left(\omega_{k+1}\right)>$ $2 \pi / j$.

End For.

$\operatorname{Return}(\mathcal{Z})$.

From our sets of initial points $\mathcal{Z}_{j}$, we obtain our approximate zeros via the routine iterate-PLM. This applies the path lifting method to $f$ for an appropriate number of steps. For simplicity, we present a scalar version here. However, there are wellknown methods for evaluating the same polynomial $f$ at $m$ different points $(m \geq d)$ in $\mathcal{O}\left(m(\log d)^{2}\right)$ arithmetic operations; refer to section 4.5 of [BM75]. When computing the complexity in Section 2.7, we assume such methods are used. Note that this algorithm can be easily implemented on either a vector or parallel computer, so that one can iterate the $d$ elements of $\mathcal{Z}_{j}$ simultaneously. Also note that if Smale's mean value conjecture holds, the extra iteration to obtain $\hat{z}$ is not needed; $z_{N}$ is good enough. See the remarks following Lemma 1.4.

\section{function scalar-iterate-PLM $\left(f, z_{0}, j, \tau\right)$}

Let $w_{0}=\left|f\left(z_{0}\right)\right| \mathrm{e}^{j \pi \mathrm{i} / 2}$.

Let $h=1 / 27$.

Let $N=\left\lfloor\frac{\log _{2}\left(\tau /\left|w_{0}\right|\right)}{\log _{2}(26 / 27)}\right\rfloor$

For $n=1$ to $N$

$$
\begin{aligned}
& \text { Let } w_{n}=(1-h) w_{n-1} . \\
& \text { Let } z_{n}=z_{n-1}-\frac{f\left(z_{n-1}\right)-w_{i}}{f^{\prime}\left(z_{n-1}\right)} .
\end{aligned}
$$

End for.

Let $\hat{z}=z_{N}-\frac{f\left(z_{N}\right)-\tau \mathrm{e}^{j \pi \mathrm{i} / 2}}{f^{\prime}\left(z_{N}\right)}$.

$\operatorname{Return}(\hat{z})$.

The next routine takes the output of iterate-PLM and uses the $\alpha$ function (defined in Lemma 1.3) to remove those elements which are not approximate zeros. Although the test $\alpha<1 / 8$ is sufficient, it is not a necessary condition. However, if the image of the initial points $\mathcal{Z}_{j}$ lie in a "good quadrant", we know by Lemma 2.5 below that we will have $\alpha<1 / 8$ for at least half of them, and so we are not in danger of discarding too many points.

\section{function select-approx-zeros $(\psi, \mathcal{Y})$}

Let $\mathcal{X}=\emptyset$.

For $j=1$ to $\#(\mathcal{Y})$

If $\alpha_{\psi}\left(y_{j}\right)<1 / 8$, then let $\mathcal{X}=\mathcal{X} \cup\left\{y_{j}\right\}$.

End For.

$\operatorname{Return}(\mathcal{X})$. 
Once we have found approximate zeros for at least half of the roots of $f_{i}$ (by applying iterate-PLM to at most 4 sets $\mathcal{Z}_{j}$ ), we further refine them by "polishing" with regular Newton's method. As in iterate-PLM, fast polynomial evaluation techniques can be used, but we present a scalar version here for simplicity.

\section{function scalar-polish-roots $\left(\psi, x_{0}, \tau\right)$}

Let $M=1+\left\lfloor\log _{2} \log _{2}\left(64 d(7 / 4)^{d}\right) / \tau\right\rfloor$.

For $n=1$ to $M$

$$
\text { Let } x_{n}=x_{n-1}-\frac{\psi\left(x_{n-1}\right)}{\psi^{\prime}\left(x_{n-1}\right)} \text {. }
$$

End For.

$\operatorname{Return}\left(x_{M}\right)$.

Finally, we remove from the set of approximations that we have found any points which approximate the same root of $\psi$. We do this by taking the approximation which minimizes $|\psi|$, and then making a pass through the rest of them and accepting only those which approximate different roots from the ones previously accepted.

\section{function weed-out-duplicates $(\psi, \mathcal{W})$}

Sort $\mathcal{W}$ so that $\left|\psi\left(w_{1}\right)\right| \leq\left|\psi\left(w_{2}\right)\right| \leq \ldots \leq\left|\psi\left(w_{n}\right)\right|$.

Let $\mathcal{V}=\left\{w_{1}\right\}$.

For $j=2$ to $\#(\mathcal{W})$

$$
\text { If }\left|w_{j}-v\right|>3\left|\psi\left(w_{j}\right)\right| /\left|\psi^{\prime}\left(w_{j}\right)\right| \text { for all } v \in \mathcal{V} \text {, then let } \mathcal{V}=\mathcal{V} \cup\left\{w_{j}\right\} .
$$

\section{End For.}

$\operatorname{Return}(\mathcal{V})$.

At this point we have found approximations to at least half of the roots of $f_{i}$. We divide them out to obtain a new polynomial $f_{i+1}$ of smaller degree, using a standard technique involving the finite Fourier matrix.

\section{function deflate $(\psi, \mathcal{V})$}

Let $n=\operatorname{deg} \psi-\# \mathcal{V}$.

Let $\omega=\mathrm{e}^{2 \pi \mathrm{i} /(n+1)}$.

Let $p(z)=\prod_{k=1}^{\# \mathcal{V}}\left(z-v_{k}\right)$.

Let $\mathcal{M}^{-1}$ be the inverse of the $n \times n$ Fourier matrix. That is, $m_{j, k}=\omega^{-j k} / n$.

Let $\mathcal{P}$ be the column vector with entries $p_{j}=\frac{\psi\left(\omega^{j}\right)}{p\left(\omega^{j}\right)}, \quad j=0, \ldots, n$.

Let $\mathcal{Q}=\mathcal{M}^{-1} \mathcal{P}$.

Let $q(z)=\sum_{j=0}^{n} q_{j} z^{j}$.

$\operatorname{Return}(q)$. 
We now state our main theorem, which essentially says that the algorithm just presented works:

THEOREM 2.1. Given a monic polynomial $\phi(z)$ of degree $d$ and $\epsilon>0$, the algorithm presented in this section will always terminate with $d$ points $\lambda_{1}, \ldots, \lambda_{d}$ which satisfy

$$
\left\|\phi(z)-\prod_{j=1}^{d}\left(z-\lambda_{j}\right)\right\|<\epsilon .
$$

For $\phi \in \mathcal{P}_{d}(1)$, the worst case arithmetic complexity of this algorithm is

$$
\mathcal{O}\left(d(\log d)^{2}|\log \epsilon|+d^{2}(\log d)^{2}\right)
$$

COROLlary 2.2. Let $\phi$ and $\epsilon$ be as in the theorem, and denote the roots of $\phi$ by $\xi_{j}$, represented with multiplicity. Then the algorithm can be used to produce the approximations $\lambda_{j}$ satisfying

$$
\left|\xi_{j}-\lambda_{j}\right|<\epsilon
$$

with a worst-case arithmetic complexity of

$$
\mathcal{O}\left(d^{2}(\log d)^{2}|\log \epsilon|+d^{2}(\log d)^{2}\right) .
$$

Proof. This is an immediate consequence of the fact that if $f$ and $g$ are in $\mathcal{P}_{d}(1)$ with $\|f-g\|<(\epsilon / 8 d)^{d}$, then the roots of $f$ and $g$ are at most $\epsilon$ apart (See Kim89b).

We prove the theorem as a series of lemmas in the following sections. There is a rough correspondence between the sections and the routines in the algorithm. Finally, we summarize all of these lemmas and give the proof in Section 2.8.

2.2. Selection of initial points. For each intermediate polynomial $f_{i}$, we need to select four sets of ( $\operatorname{deg} f_{i}$ ) points at which to begin our iteration. These must be chosen so that the elements of each set map very near the same point in the target space, and that the images of elements in successive groups are approximately $\frac{1}{4}$-turn apart. This can be accomplished either by evaluating $f_{i}$ at a large number of points spaced around a circle in the source space, and then selecting from those, or by taking a much smaller number (perhaps as few as $4 d$ ) of points and adjusting them with either a standard or modified Newton's method. The arithmetic complexity of either comes out much the same; we opt for the former method because of its conceptual simplicity.

The following lemma gives us bounds on how much the argument in the target space can vary between points around a circle containing all the roots in the source space. Using this, we see how many points are required to obtain our "good" points.

LEMma 2.3. Suppose all of the roots $\zeta$ of a polynomial $f$ of degree d lie in $\mathbb{D}_{R}(0)$, and let

$$
\omega_{m}=2 R \mathrm{e}^{\frac{2 \pi \mathrm{i} m}{n d}} .
$$


Then

$$
\left|\arg \frac{f\left(\omega_{m+1}\right)}{f\left(\omega_{m}\right)}\right| \leq \frac{4 \pi}{n}
$$

Proof. This argument appears in [Ren87](Lemma 7.1), although in a somewhat different form. We present an adapted version here. The idea is quite simple: since

$$
\arg \frac{f\left(\omega_{m+1}\right)}{f\left(\omega_{m}\right)}=\arg \frac{\prod_{i=1}^{d}\left(\omega_{m+1}-\zeta_{i}\right)}{\prod_{i=1}^{d}\left(\omega_{m}-\zeta_{i}\right)}=\sum_{i=1}^{d} \arg \frac{\omega_{m+1}-\zeta_{i}}{\omega_{m}-\zeta_{i}},
$$

we merely bound the angles in the source space and add them up.

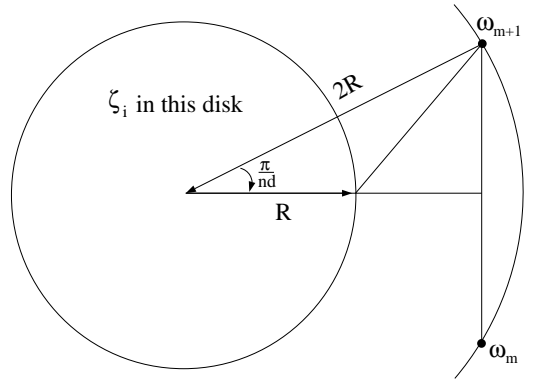

FIG. 2.1. Calculation of the upper bound on the variation of argument in the target space. This picture is in the source space.

Note that each root $\left|\zeta_{i}\right|<R$ and $\left|\omega_{m}\right|=2 R$, so

$$
\begin{aligned}
\left|\arg \frac{\omega_{m+1}-\zeta_{i}}{\omega_{m}-\zeta_{i}}\right| & \leq 2 \arctan \frac{\left|\omega_{m+1}-\omega_{m}\right| / 2}{2 R \cos \frac{\pi}{n d}-R} \\
& \leq 2 \arctan \frac{2 R \sin \frac{\pi}{n d}}{2 R\left(\cos \frac{\pi}{n d}-\frac{1}{2}\right)} \\
& \leq \frac{4 \pi}{n d} .
\end{aligned}
$$

Refer to Figure 2.1. Summing the $d$ terms gives the result.

From Theorem 1.5, for $h=1 / 27$ we require that our initial points be within $\sin ^{-1}(h / 2)<\pi / 169$ of the central ray, so we start with $676 d$ points equally spaced around the circle of radius $3 / 2$. We then evaluate the polynomial at each of them, and select four sets of $d$ points whose arguments are closest to $0, \frac{\pi}{2}, \pi$, and $\frac{3 \pi}{2}$, respectively. For each of these, we take the initial target point $w_{0}$ to be the projection of its image onto the real or imaginary axis.

REMARK. Some amount of computation can be saved if we use the same $w_{0}$ for all $d$ elements of a given set of initial points, rather than just points with the same argument. This would make the computation of the $w_{n}$ a scalar rather than a vector operation, that is, $w_{n}$ would only need to be computed once for each group of $d$ points. However, in order to do this, we must ensure that the images of each $z_{j}$ in the same set 
have approximately the same modulus as well as argument. This is best accomplished using some sort of Newton's method.

If an initial Newton's method is used, one can also choose a much smaller number of trial points $\omega_{i}$. Such a method should converge well, since all the critical points of $f(z)-w_{0}$ are inside the $\mathbb{D}_{R}$, while the roots of $f(z)-w_{0}$ and the $\omega_{i}$ are well outside.

2.3. Iteration of the path lifting method. In this section, we analyze the behavior of applying the path lifting method to a single well-chosen initial value $z_{0}$.

We first prove the theorem as promised in Section 1.3, and then we show that after a specified number of iterations, the result will be an approximate zero. Before doing this, we shall state the relevant special case of Theorem 3.2 of [Kim88, which gives a lower bound on how far a point moves under Newton's method.

LEMMA 2.4. Let $\hat{z}=z-g(z) / g^{\prime}(z)$, with $r=|g(z)| / R_{g}(z)<0.148$, where $R_{g}(z)$ is the radius of convergence of $g_{z}^{-1}$ as a power series based at $z$. Then

$$
\left|\frac{g(\hat{z})}{g(z)}\right| \leq B(r) \quad \text { where } \quad B(r)=2 r \frac{(1+r)^{3}}{(1-r)^{5}}
$$

Given this lemma, the proof of Theorem 1.5 is quite simple. Note that each step of the iteration in Theorem 1.5 corresponds to Newton's method applied to $f(z)-w_{n+1}$ at $z_{n-1}$.

THEOREM 1.5. Suppose that the branch of the inverse $f_{z_{0}}^{-1}$ is analytic on a wedge $\mathcal{W}_{A, w_{0}}$, with $0 \leq A \leq \pi / 2$, and let $h \leq \frac{\sin A}{19}$. Suppose also that $\left|f\left(z_{0}\right)-w_{0}\right|<h\left|w_{0}\right| / 2$, and define

$$
w_{n}=(1-h)^{n} w_{0}, \quad z_{n+1}=z_{n}-\frac{f\left(z_{n}\right)-w_{n+1}}{f^{\prime}\left(z_{n}\right)} .
$$

Then $\left|f\left(z_{n}\right)-w_{n}\right| \leq h\left|w_{n}\right| / 2$ and $z_{n+1} \in f_{z_{0}}^{-1}\left(\mathcal{W}_{A, w_{n}}\right)$.

Proof. We shall prove this by induction. All that is required is to establish the conclusion, given that $\left|f\left(z_{n-1}\right)-w_{n-1}\right|<h\left|w_{n-1}\right| / 2$.

Note that

$$
\left|f\left(z_{n-1}\right)-w_{n}\right| \leq\left|f\left(z_{n-1}\right)-w_{n-1}\right|+\left|w_{n-1}-w_{n}\right| \leq 3 h\left|w_{n-1}\right| / 2
$$

and that

$$
R_{f-w_{n}}\left(z_{n-1}\right)=R_{f}\left(z_{n-1}\right) \geq\left|w_{n}\right| \sin A-h\left|w_{n}\right| / 2 .
$$

Since $h \leq \frac{\sin A}{19}$, we can apply Lemma 2.4 with $g(z)=f(z)-w_{n}$ and $r=3 / 37$ to obtain

$$
\left|\frac{f\left(z_{n}\right)-w_{n}}{w_{n}}\right|=\left|\frac{f\left(z_{n}\right)-w_{n}}{f\left(z_{n-1}\right)-w_{n}}\right| \cdot\left|\frac{f\left(z_{n-1}\right)-w_{n}}{w_{n-1}(1-h)}\right| \leq B(r) \frac{3 h}{2(1-h)} .
$$

Because $B(3 / 37)<(1-h) / 3$, we have our conclusion. 
REMARK. The denominator of 19 in the upper bound on $h$ can be relaxed only slightly in this proof. If we take $h \leq \frac{\sin A}{k(A)}$, then $k(A)$ is a monotonically increasing function, with $18.3096 \leq k(A) \leq 18.895$. Also, if $A>\pi / 2$, we can take $h=1 / 19$.

We apply the path lifting method until we have an approximate zero, at which time we can use the standard Newton iteration. Since we are interested in finding $\tau$-roots of $f$, we stop the iteration once we have an approximate zero for $f-\tau \frac{w_{0}}{\left|w_{0}\right|}$. Note that, as discussed in Section 2.1, this translation is necessary, since there are no approximate zeros in a neighborhood of a multiple root. Having approximate zeros for the perturbed polynomial gives us the following bound on the number of iterations required, and enables the weeding out of duplicates.

LEMmA 2.5. Let $h \leq \frac{\sin A}{19}$, with $w_{i}$ and $z_{i}$ as in Theorem 1.5. If

$$
N=\left\lfloor\frac{\log _{2}\left(\tau /\left|w_{0}\right|\right)}{\log _{2}(1-h)}\right\rfloor,
$$

then $z_{N}$ is an approximate zero for $f-\tau \frac{w_{0}}{\left|w_{0}\right|}$. Furthermore, if $\hat{z}=z_{N}-\frac{f\left(z_{N}\right)-\tau \frac{w_{0}}{\left|w_{0}\right|}}{f^{\prime}\left(z_{N}\right)}$ then we have $\alpha(\hat{z}) \leq 1 / 8$.

Proof. For notational convenience, set $\vec{\tau}=\tau \frac{w_{0}}{\left|w_{0}\right|}$.

By assumption, $f_{z_{0}}^{-1}$ is analytic on the wedge $\mathcal{W}_{A, w_{0}}$, and the initial point $z_{0}$ is close enough to $w_{0}$ that we can apply Theorem 1.5. Note that if $N$ is as specified, we have

$$
\tau \leq\left|w_{N}\right| \leq \frac{\tau}{1-h}
$$

since $\left|w_{N}\right|=(1-h)^{N}\left|w_{0}\right|$ by definition. Also, $\left|f\left(z_{N}\right)-w_{N}\right| \leq h\left|w_{N}\right| / 2$. Refer to Figure 2.2.

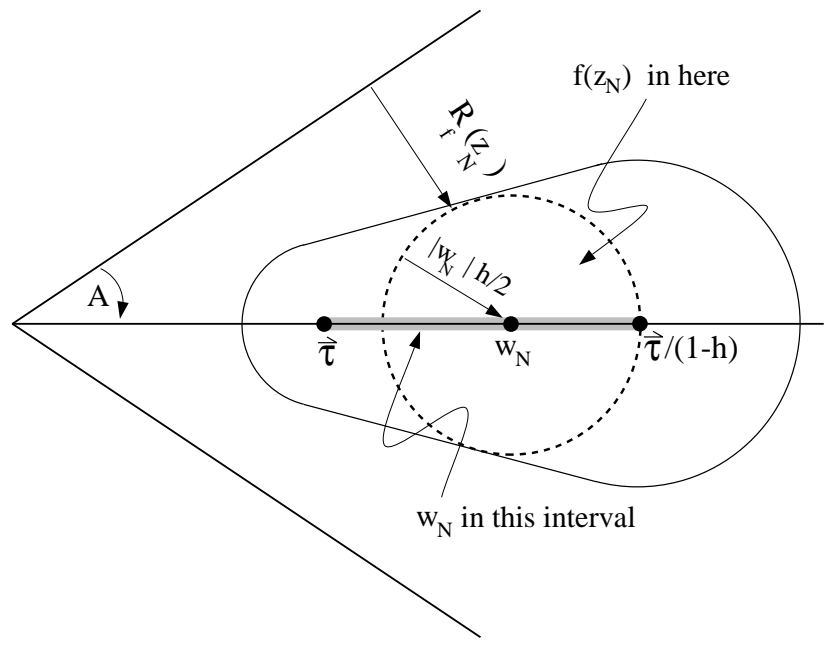

FIG. 2.2. The locations of $w_{N}$ and $f\left(z_{N}\right)$ in the target space when $z_{0}$ is in a "good quadrant".

In order to conclude that $z_{N}$ is an approximate zero, we need to show that we have $\left|f\left(z_{N}\right)-\vec{\tau}\right| / R_{f}\left(z_{N}\right)<1 / 10$, where $R_{f}\left(z_{N}\right)$ is the radius of convergence of $f_{z_{N}}^{-1}$. 
Consider $f\left(z_{N}\right)$ in some circle of radius $h|w| / 2$, where $\arg w=\arg w_{0}$, and $|w|>\tau$. Then we have

$$
\frac{\left|f\left(z_{N}\right)-\vec{\tau}\right|}{R_{f}\left(z_{N}\right)} \leq \frac{|w|+h|w| / 2-\tau}{|w| \sin A-h|w| / 2}=\frac{2+h}{2 \sin A-h}-\frac{\tau}{|w|(\sin A-h / 2)} .
$$

The maximum of this quantity occurs when $|w|$ is as large as possible, which in our case is $|w|=\tau /(1-h)$. Hence

$$
\frac{\left|f\left(z_{N}\right)-\vec{\tau}\right|}{R_{f}\left(z_{N}\right)} \leq \frac{3 h}{2 \sin A-h} \leq 3 / 37<1 / 10 .
$$

By Lemma 1.4, $z_{N}$ is an approximate zero of $f(z)-\vec{\tau}$.

In order to ensure that $\alpha<1 / 8$, we need to apply Newton's method once. Set

$$
\hat{z}=z_{N}-\frac{f\left(z_{N}\right)-\vec{\tau}}{f^{\prime}\left(z_{N}\right)}
$$

Then this point satisfies

$$
\frac{|f(\hat{z})-\vec{\tau}|}{\left|f\left(z_{N}\right)-\vec{\tau}\right|} \leq B(3 / 37) \leq 0.31271
$$

and so

$$
|f(\hat{z})-\vec{\tau}| \leq 0.31271\left(\left|f\left(z_{N}\right)-w_{N}\right|+\left|w_{N}\right|-\tau\right) \leq 0.31271 \frac{3 h \tau}{2(1-h)} \leq \frac{\tau \sin A}{39}
$$

This gives us

$$
\frac{|f(\hat{z})-\vec{\tau}|}{R_{f}(\hat{z})} \leq \frac{\tau \sin A / 39}{\tau \sin A-\tau \sin A / 39}=1 / 38 .
$$

As a consequence of Lemma 1.4, we have $\alpha(\hat{z})<1 / 8$.

2.4. Refinement of the root approximations. The routine iterate-PLM outputs a set of points $\mathcal{Y}=\left\{y_{1}, y_{2}, \ldots, y_{d}\right\}$ which may be approximate zeros for $\psi(z)=$ $f(z)-\vec{\tau}$. We can use $\alpha_{\psi}$ (see Lemma 1.3) to choose those $y_{i}$ that are indeed approximate zeros; we discard those $y_{i}$ for which $\alpha_{\psi}(z)>1 / 8$. As a consequence of Lemma 1.4 and Lemma 2.5, those $y_{i}$ which started in the "good sector" will not be discarded.

In addition, we also want to ensure that we approximate each root only once (counted with multiplicity). Lemma 2.7 gives us conditions which allow us to weed out any duplicates; the proof relies on a variant of the Koebe Distortion Theorem which we quote here from Kim88, Lemma 3.3.

Lemma 2.6. Let $g$ be univalent on $\mathbb{D}_{R}(z)$. Then for any $s<1$, we have

$$
\mathbb{D}_{t R}(g(z)) \subset g\left(\mathbb{D}_{s R}(z)\right) \subset \mathbb{D}_{u R}(g(z)),
$$

where $t=s\left|g^{\prime}(z)\right| /(1+s)^{2}$ and $u=s\left|g^{\prime}(z)\right| /(1-s)^{2}$. 
LEMMA 2.7. Suppose that $y_{1}$ and $y_{2}$ are approximate zeros of $\psi(z)$, with $\left|\psi\left(y_{1}\right)\right| \geq$ $\left|\psi\left(y_{2}\right)\right|$, and $R_{\psi}\left(y_{j}\right) \geq 12 \psi\left(y_{j}\right)$, where $R_{\psi}\left(y_{j}\right)$ is the radius of convergence of $\psi_{y_{j}}^{-1}$. Then $y_{1}$ and $y_{2}$ approximate the same simple root $\xi$ of $\psi$ if and only if

$$
\left|y_{1}-y_{2}\right|<\left|\frac{3 \psi\left(y_{1}\right)}{\psi^{\prime}\left(y_{1}\right)}\right| \text {. }
$$

Proof. If $y_{1}$ and $y_{2}$ approximate the same simple root, then we have $\psi_{y_{1}}^{-1}=\psi_{y_{2}}^{-1}$. Since $\left|\psi\left(y_{1}\right)-\psi\left(y_{2}\right)\right| \leq 2\left|\psi\left(y_{1}\right)\right|$, we have $\psi\left(y_{2}\right) \in \mathbb{D}_{2\left|\psi\left(y_{1}\right)\right|}\left(\psi\left(y_{1}\right)\right)$. Thus we can apply Lemma 2.6 with $g=\psi^{-1}$, which is univalent on the disk of radius $R=12\left|\psi\left(y_{1}\right)\right|$. Taking $s=1 / 6$, we have $\psi^{-1}\left(\mathbb{D}_{2\left|\psi\left(y_{1}\right)\right|}\left(\psi\left(y_{1}\right)\right)\right)$ contained in the disk of radius $\frac{72\left|\psi\left(y_{1}\right)\right|}{25\left|\psi^{\prime}\left(y_{1}\right)\right|}$ about $y_{1}$, so $\left|y_{1}-y_{2}\right|<\frac{3\left|\psi\left(y_{1}\right)\right|}{\left|\psi^{\prime}\left(y_{1}\right)\right|}$.

For the other direction, we apply the Koebe $\frac{1}{4}$-Theorem (or Lemma 2.6 with $s=$ 1 ; it is the same thing) to see that $\psi_{y_{1}}^{-1}\left(\mathbb{D}_{12\left|\psi\left(y_{1}\right)\right|}\left(\psi\left(y_{1}\right)\right)\right)$ contains the disk of radius $3\left|\psi\left(y_{1}\right)\right| /\left|\psi^{\prime}\left(y_{1}\right)\right|$ about $y_{1}$. Thus, if the distance between $y_{1}$ and $y_{2}$ is less than this amount, we must have $\psi_{y_{1}}^{-1}\left(\psi\left(y_{2}\right)\right)=y_{2}$, that is, $y_{1}$ and $y_{2}$ approximate the same root of $\psi$.

REMARK. Note that $\alpha_{\psi}(z)<1 / 8$ is not sufficient to imply that the hypotheses of Lemma 2.7 are satisfied, since this only gives $R_{\psi}(z) \geq 4|\psi(z)| / 3$. However, if Newton's method is applied to such a point at least 3 times, the value of $|\psi|$ will decrease by at least $1 / 128$, and so the lemma can be applied. Since we need to "polish" the approximations with Newton's method in order to control the error in the deflation, we do that before weeding out the duplicates. The total number of iterations of Newton's method required is calculated in Section 2.6, but it is greater than 3 in all cases. In practice, one should probably perform the weeding in the routine select-approx-zeros, in order to avoid polishing points which will be discarded later.

2.5. Deflation of intermediate polynomials. Here we compute an explicit bound on the error introduced by the deflation step. We start with a polynomial $\psi$ which has roots $\left\{\xi_{j}\right\}_{j=1, \ldots, d}$, and a set of approximations to these roots which we denote by $\left\{v_{k}\right\}_{k=1, \ldots, n}$ with $n \leq d$. We then use polynomial interpolation via the discrete Fourier matrix to obtain a polynomial $q$ of degree $d-n$ so that

$$
\psi(z) \approx p(z) q(z), \quad \text { where } \quad p(z)=\prod_{k=1}^{n}\left(z-v_{k}\right) .
$$

In this section, we estimate $\|\psi(z)-p(z) q(z)\|$, as well as the accumulated error in repeating this process until $q(z)$ is a constant. Recall that the finite Fourier matrix $\mathcal{M}$ has as its $j, k^{t h}$ entry the $j k^{t h}$ power of a primitive $d+1^{\text {st }}$-root of unity. That is,

$$
m_{j, k}=\omega^{j k}=\mathrm{e}^{2 \pi \mathrm{i} j k /(d+1)} \quad j, k=0, \ldots, d
$$

Then one can readily see that if $f(z)=\sum_{j=0}^{d} a_{j} z^{j}$ and $\mathcal{A}$ is the column vector of the coefficients of $f$, then the product $\mathcal{M A}$ will be the vector $\mathcal{B}$ whose $j^{\text {th }}$ entry is the 
value of $f\left(\omega^{j}\right)$. Also, given the values of $f$ evaluated at the powers of $\omega$, we can easily compute the coefficients of $f$ as the product $\mathcal{M}^{-1} \mathcal{B}$.

Our first lemma gives us estimates on the size of the error caused by a single deflation. As is common with this sort of thing, the proof is neither terribly entertaining or enlightening.

LEMMA 2.8. Suppose $\psi(z)=\prod_{j=1}^{d}\left(z-\xi_{j}\right)$, with $\left|\xi_{j}\right|<3 / 4$, factors as $\psi(z)=$ $P(z) Q(z)$, where $\operatorname{deg}(P)=n<d$ and $\operatorname{deg}(Q)=m=d-n$. Let $p(z)=\prod_{j=1}^{n}\left(z-v_{j}\right)$, with $\left|\xi_{j}-v_{j}\right|<\delta<\frac{1}{8 d^{2}}$, and define $q(z)$ and $r(z)$ by $\psi(z)=p(z) q(z)+r(z)$, where $q$ is found by polynomial interpolation as described above. Then

$$
\begin{aligned}
\|P(z)-p(z)\| & <8 n \delta(7 / 4)^{n} \\
\|Q(z)-q(z)\| & <8 m \delta(7 / 4)^{m} \\
\|r(z)\| & <8 d \delta(7 / 4)^{d} .
\end{aligned}
$$

Proof. Let $\omega=\mathrm{e}^{2 \pi \mathrm{i} /(n+1)}$, and let $\mathcal{B}$ be the vector with entries $b_{j}=P\left(\omega^{j}\right)-p\left(\omega^{j}\right)$, for $0 \leq j \leq n$. Note that

$$
\begin{aligned}
b_{j} & =\prod_{k=1}^{n}\left(\omega^{j}-\xi_{k}\right)-\prod_{k=1}^{n}\left(\omega^{j}-v_{k}\right) \\
& =\left(\prod_{k=1}^{n}\left(\omega^{j}-\xi_{k}\right)\right)\left(1-\prod_{k=1}^{n} \frac{\omega^{j}-v_{k}}{\omega^{j}-\xi_{k}}\right) \\
& =\left(\prod_{k=1}^{n}\left(\omega^{j}-\xi_{k}\right)\right)\left(1-\prod_{k=1}^{n}\left(1+\frac{\xi_{k}-v_{k}}{\omega^{j}-\xi_{k}}\right)\right) .
\end{aligned}
$$

Also note that

$$
\frac{1}{4}<\left|\omega^{j}-\xi_{k}\right|<\frac{7}{4} \quad \text { and } \quad\left|\frac{\xi_{k}-v_{k}}{\omega^{j}-\xi_{k}}\right| \leq 4 \delta
$$

Since $4 \delta \leq \frac{1}{2 d^{2}}<\frac{1}{2 n^{2}}$, we have $(1+4 \delta)^{n} \leq 8 n \delta+1$, and so

$$
\left|b_{j}\right|<(7 / 4)^{n}\left((1+4 \delta)^{n}-1\right)<8 n \delta(7 / 4)^{n} .
$$

Thus

$$
\|P(z)-p(z)\|=\left\|\mathcal{M}^{-1} \mathcal{B}\right\| \leq\|\mathcal{B}\|<8 n \delta(7 / 4)^{n}
$$

We have a similar computation for the bound on $\|Q-q\|$ : Let $\eta=\mathrm{e}^{2 \pi \mathrm{i} /(m+1)}$ and let $\mathcal{C}$ be the vector with entries $c_{j}=Q\left(\eta^{j}\right)-q\left(\eta^{j}\right), j=0, \ldots, m$. Then

$$
c_{j}=\frac{\psi\left(\eta^{j}\right)}{P\left(\eta^{j}\right)}-\frac{\psi\left(\eta^{j}\right)}{p\left(\eta^{j}\right)}=\frac{\psi\left(\eta^{j}\right)}{P\left(\eta^{j}\right)}\left(1-\frac{P\left(\eta^{j}\right)}{p\left(\eta^{j}\right)}\right)=Q\left(\eta^{j}\right)\left(1-\prod_{k=1}^{m} \frac{\eta^{j}-v_{k}}{\eta^{j}-\xi_{k}}\right) .
$$


As in the case of $P(z)-p(z)$, we obtain

$$
\|Q(z)-q(z)\|=\left\|\mathcal{M}^{-1} \mathcal{C}\right\| \leq\|\mathcal{C}\|<8 m \delta(7 / 4)^{m} .
$$

Finally, for the bound on $\|r(z)\|$, note that $r(z)=P(z) Q(z)-p(z) q(z)$. If we write $r(z)=\sum_{j=0}^{d} r_{j} z^{j}, P(z)=\sum_{j=0}^{d} P_{j} z^{j}$, and so on, then we have

$$
r_{j}=\sum_{k=0}^{j} P_{j-k} Q_{k}-\sum_{k=0}^{j} p_{j-k} q_{k}=\sum_{k=0}^{j}\left(q_{k}\left(P_{j-k}-p_{j-k}\right)-P_{j-k}\left(Q_{k}-q_{k}\right)\right) .
$$

Since $\left|\xi_{j}\right|<3 / 4$, we have the following crude bounds on the coefficients of $P$ and $q$ :

$$
\left|P_{j-k}\right| \leq(7 / 4)^{n} \quad \text { and } \quad\left|q_{j}\right| \leq(7 / 4)^{m} .
$$

Combining this with the bounds on $\|P-p\|$ and $\|Q-q\|$, we get

$$
\begin{aligned}
\left|r_{j}\right| & \leq \sum_{k=0}^{j}\left(8 m \delta(7 / 4)^{m}(7 / 4)^{n}+8 n \delta(7 / 4)^{n}(7 / 4)^{m}\right) \\
& \leq 8 j d \delta(7 / 4)^{d} \\
& \leq 8 d^{2} \delta(7 / 4)^{d} .
\end{aligned}
$$

Now that we have bounds on the error in one step of deflation, we can bound the error introduced by repeated deflation. We assume that our initial polynomial $f$ has roots in $\mathbb{D}_{1 / 2}$ so that we can ensure that the roots of the subsequent polynomials $f_{k}$ remain in $\mathbb{D}_{3 / 4}$ as required by Lemma 2.8 .

Lemma 2.9. Suppose $f(z)=\prod_{j=1}^{d}\left(z-\zeta_{j}\right)$, with $\left|\zeta_{j}\right|<1 / 2$. Let $f_{0}=f$, and define

$$
f_{k}(z)=p_{k+1}(z) f_{k+1}(z)+r_{k+1}(z)
$$

for $0<k<m-1$, where $p_{j}, f_{j}$, and $r_{j}$ are determined by polynomial interpolation as in Lemma 2.8, with $f_{m}(z)=1$. Suppose also that $\operatorname{deg} r_{k+1}<\operatorname{deg} f_{k}=d_{k}$, and that $\left\|r_{k}\right\|<\min \left(\frac{1}{4(4 d)^{d}}, \mu(4 / 7)^{d+3}\right)$. Then

$$
\left\|f-p_{1} p_{2} \cdots p_{m}\right\|<\mu .
$$

Furthermore, if we have $d_{k} \leq d_{k-1} / 2$ and $\mu \leq(7 / 16)^{d}$, we need only require that $\left\|r_{k}\right\|<\mu(4 / 7)^{d+3}$.

Proof. Note that $f-p_{1} p_{2} \cdots p_{m}=p_{1} \cdots p_{m} r_{m-1}+\ldots+p_{1} r_{2}+r_{1}$, and so

$$
\left\|f-p_{1} p_{2} \cdots p_{m}\right\| \leq\left\|p_{1} \cdots p_{m-1}\right\|\left\|r_{m-1}\right\|+\ldots+\left\|p_{1}\right\|\left\|r_{2}\right\|+\left\|r_{1}\right\| .
$$

First, we show that $\left\|p_{1} \cdots p_{k}\right\| \leq(7 / 4)^{\operatorname{deg} p_{1} \cdots p_{k}}$. We shall use induction to show that the roots $p_{1} \cdots p_{k}$ are always in the circle of radius $\frac{1}{2}+\frac{k}{4 d}<3 / 4$. On the circle $|z|=\frac{1}{2}+\frac{k}{4 d}$, we have

$$
\left|f_{k-1}(z)-p_{k}(z) f_{k}(z)\right| \leq\left\|r_{k}\right\| \sum_{j=0}^{\operatorname{deg} r_{k}}|z|^{j} \leq \frac{d}{(4 d)^{d}(2 d-k)} \leq \frac{1}{(4 d)^{d}} \leq\left|f_{k-1}(z)\right|,
$$


and so by Rouché's Theorem the roots of $p_{k} f_{k}$ lie inside $\mathbb{D}_{\frac{1}{2}+\frac{k}{4 d}}$. Thus the roots of $p_{1} p_{2} \cdots p_{k}$ also lie in this disk, and so the coefficients are less than $(7 / 4)^{\operatorname{deg} p_{1} \cdots p_{k}}$.

Since $\operatorname{deg} p_{1} p_{2} \cdots p_{k}<d-(m-k)$, we can conclude that

$$
\begin{aligned}
\sum_{k=1}^{m-1}\left\|p_{1} p_{2} \cdots p_{k}\right\|\left\|r_{k+1}\right\| & \leq \sum_{k=1}^{m-1}(7 / 4)^{d-(m-k)}(4 / 7)^{d+3} \mu \\
& \leq \frac{4}{3}(7 / 4)^{d+2}(4 / 7)^{d+3} \mu \\
& <\mu .
\end{aligned}
$$

If we require that $d_{k} \leq d / 2^{k}$, that is, we find at least half of the roots at each step, then we can relax the restriction on $\left\|r_{k}\right\|$ somewhat. If we have $\left\|r_{k}\right\| \leq \frac{1}{4 \cdot 4^{d}}$, then we can use Rouché's theorem as before to show that the roots of $p_{k} f_{k}$ are in the disk of radius $C_{k}=\frac{1}{2}+\frac{1}{2} \sum_{n=1}^{k} 2^{-2^{k}}<3 / 4$. Note that for $|z|=C_{k}$, we have

$$
\left|f_{k-1}(z)\right| \geq\left(C_{k}-C_{k-1}\right)^{d_{k-1}} \geq\left(\frac{1}{2 \cdot 2^{2^{k}}}\right)^{d / 2^{k-1}} \geq 1 / 4^{d}
$$

Applying almost the same argument as before, we have

$$
\left|f_{k-1}(z)-p_{k}(z) f_{k}(z)\right| \leq\left\|r_{k}\right\| \sum_{j=0}^{\operatorname{deg} r_{k}}|z|^{j} \leq \frac{1}{4^{d}} \leq\left|f_{k-1}(z)\right| .
$$

Since $\left\|r_{k}\right\|<\mu(4 / 7)^{d+3}<\frac{1}{4 \cdot 4^{d}}$, we are done.

2.6. Controlling the error. In this section, we compute the size of $\tau$ that we can use to ensure we have an $\epsilon$-factorization of $\psi$. The following very simple proposition shows that if the norms of two polynomials are close, so are the norms of the rescaled versions. This gives us the relationship between $\epsilon$ and the number $\mu$ used in Lemma 2.9.

Proposition 2.10. Suppose that $\phi$ is a monic polynomial with all its roots in $\mathbb{D}_{R}$, so that the roots of $f(z)=(2 R)^{-d} \phi(2 R z)$ are in $\mathbb{D}_{1 / 2}$. Then if $\|f-p\| \leq \epsilon$, we have

$$
\left\|\phi(z)-(2 R)^{d} p\left(\frac{z}{2 R}\right)\right\| \leq(2 R)^{d} \epsilon .
$$

Proof. Let $f(z)=\sum a_{j} z^{j}$ and $p(z)=\sum b_{j} z^{j}$. Then $\phi(z)=\sum(2 R)^{d-j} a_{j} z^{j}$ and $(2 R)^{d} p\left(\frac{z}{2 R}\right)=\sum(2 R)^{d-j} b_{j} z^{j}$. Since $\max \left|\sum(2 R)^{d-j}\left(a_{j}-b_{j}\right)\right| \leq(2 R)^{d}\left|a_{j}-b_{j}\right|$, we have our claim.

Our input polynomial $\phi$ is in $\mathcal{P}_{d}(1)$ and $f$ is be the rescaled polynomial as in the previous proposition, so $R=2$. Then an $\epsilon$-factorization of $\phi$ corresponds to an $\epsilon / 4^{d}$ factorization of $f$, so we take $\mu=\epsilon / 4^{d}$.

In order to properly approximate the roots of $f$, we need to ensure that the remainder $r_{k}$ (as in Lemma 2.9) at the $k^{t h}$ step satisfies $\left\|r_{k}\right\|<2 \tau$, where $\tau=(4 / 7)^{d+3} \mu / 2=$ $32 \epsilon / 7^{d+3}$. 
At each stage, we translate $f_{k}$ by $\tau$, and ensure that the error introduced by the deflation of the translated polynomial is no more than $\tau$. By Lemma 2.8, we need the root distance between translated polynomial and the deflated polynomial to satisfy

$$
\delta \leq \frac{\tau}{8 d}\left(\frac{4}{7}\right)^{d}
$$

Then we will have

$$
\left\|r_{k}\right\|=\left\|f_{k-1}-p_{k} f_{k}\right\| \leq\left\|\left(f_{k-1}-\vec{\tau}\right)-p_{k} f_{k}\right\|+\left\|\left(f_{k-1}-\vec{\tau}\right)-f_{k-1}\right\| \leq \tau+\tau .
$$

In order to achieve the root distance less than $\delta$, we apply Newton's method to the approximate zeros found by the routine iterate-PLM (see Lemma 2.5). Since each point $z$ is an approximate zero to the root $\xi$ of the translated polynomial, we have by Definition 1.2

$$
\delta \leq 8\left(\frac{1}{2}\right)^{2^{n}}|z-\xi| .
$$

Thus, iterating Newton's method $\log _{2} \log _{2}(8 / \delta)$ times, as is done in polish-roots, will give the desired result.

2.7. Arithmetic complexity. In this section we count the number of arithmetic operations involved in using the algorithm to obtain an $\epsilon$-factorization of a polynomial in $\mathcal{P}_{d}(1)$.

In the main routine, we rescale the polynomial and then invoke get-half-rootsand-deflate at most $\log _{2} d$ times, since at least half of the roots are found in each call.

- The cost of rescaling is $2 d$ multiplications.

- Each call to get-half-roots-and-deflate calls choose-4d-good-initial-points once, and makes at most 4 calls to each of iterate-PLM, select-approx-zeros, polish-roots, and weed-out-duplicates, and one call to deflate. As before, we denote the degree of the input polynomial by $d$ and the degree of the $k^{\text {th }}$ intermediate polynomial by $d_{k}$.

- The routine choose-4d-good-initial-points involves evaluation of $f_{k}$ at $676 d_{k}$ points, and $676 d_{k}$ comparisons. Since we can evaluate $f_{k}$ at $m$ points with a cost of $\mathcal{O}\left(m\left(\log d_{k}\right)^{2}\right)$ operations (see BM75), this gives a total of $\mathcal{O}\left(d_{k}\left(\log d_{k}\right)^{2}\right)$ operations.

- For iterate-PLM, each iteration evaluates $z_{n}-\frac{f\left(z_{n}\right)-w_{n+1}}{f^{\prime}\left(z_{n}\right)}$ at $d_{k}$ points, which costs $\mathcal{O}\left(d_{k}\left(\log d_{k}\right)^{2}\right)$. This is done $N$ times, where $N<27 \log \left(\frac{\left|w_{0}\right|}{\tau}\right)$. Note that since the roots of $f$ are in $\mathbb{D}_{3 / 4}$ and $\left|z_{0}\right|=3 / 2$, we have $\left|w_{0}\right|=\left|f_{k}\left(z_{0}\right)\right| \leq(9 / 4)^{d_{k}}$. Since $\tau=32 \epsilon / 7^{d+3}$, we have $N=C_{1}(d+|\log \epsilon|)$ for some constant $C_{1}$. This gives a total of

$$
\mathcal{O}\left((d+|\log \epsilon|)\left(d_{k}\left(\log d_{k}\right)^{2}\right)\right)
$$

operations. 
- For select-approx-zeros, we need to evaluate $\alpha$ at $d_{k}$ points, which requires evaluation of all the derivatives of $f$. This requires $\mathcal{O}\left(d_{k}^{2}\left(\log d_{k}\right)^{2}\right)$ operations.

- The routine polish-roots performs $M$ iterations of Newton's method at $d_{k}$ points. Since $M=C_{2}(\log d+\log |\log \epsilon|)$ for some constant $C_{2}$, we have a total operation count of

$$
\mathcal{O}\left(\left(d_{k}\left(\log d_{k}\right)^{2}\right)(\log d+\log |\log \epsilon|)\right)
$$

○ Weed-out-duplicates requires a sort of at most $d_{k}$ points, which $\operatorname{costs} \mathcal{O}\left(d_{k} \log d_{k}\right)$, and evaluation of $f$ and $f^{\prime}$ at $d_{k}$ points. Thus the total cost of this routine is $\mathcal{O}\left(d_{k}\left(\log d_{k}\right)^{2}\right)$.

○ Lastly, deflate $\operatorname{costs} \mathcal{O}\left(d_{k}\left(\log d_{k}\right)^{2}\right)$ operations.

Thus, the overall cost of each call to get-half-roots-and-deflate is dominated by that of iterate-PLM, and is at most $\mathcal{O}\left(d_{k}\left(\log d_{k}\right)^{2}(d+|\log \epsilon|)\right)$ operations. Since $d_{k+1} \leq d_{k} / 2$, we have a total cost of at most

$$
\mathcal{O}\left(d^{2}(\log d)^{2}+d(\log d)^{2}|\log \epsilon|\right)
$$

operations to obtain an $\epsilon$-factorization of the polynomial.

2.8. Summary and proof of main theorem. At this point, we have actually already proven Theorem 2.1, but we would like to tie together the various steps involved. Just to refresh your memory, this theorem says, in essence, that our algorithm always produces an $\epsilon$-factorization with the stated complexity.

Recall that the algorithm performs the approximate factorization in stages using the routine get-half-roots-and-deflate; at the $k^{\text {th }}$ step, we produce a function $f_{k}$ and sets of approximations $\Lambda_{j}$ so that

$$
f(z) \approx f_{k}(z) \prod_{\lambda_{i} \in \Lambda}\left(z-\lambda_{i}\right)
$$

where $\Lambda=\bigcup_{j \leq k} \Lambda_{j}$. In order to prove Theorem 2.1, we need to show that the number of approximations found at the $k^{t h}$ stage $\left(\# \Lambda_{k}\right)$ is at least $\left(\operatorname{deg} f_{k-1}\right) / 2$, and that $\| f(z)-$ $f_{k}(z) \prod_{\lambda_{i} \in \Lambda}\left(z-\lambda_{i}\right) \| \leq \epsilon / 4^{d}$. Given this, the complexity calculation in the previous section will apply, and we shall have the result.

First, note that as a consequence of Lemma 1.6, there will always be a quarterplane in the target space on which there at least $d_{k} / 2$ branches of $f_{k}^{-1}$ are defined. This means that if we start with $d_{k}$ points $z_{j}$ which are well-spaced in the source space (so that each sheet in the target space is represented), then for at least half of them there will be a branch of the the inverse $f_{z_{j}}^{-1}$ which is defined on the entire quadrant, and $f_{z_{i}}^{-1} \neq f_{z_{j}}^{-1}$. For these points $z_{j}$, if we ensure that $f\left(z_{j}\right)$ is close enough to the center line of the quadrant, at least half of them will satisfy the hypothesis of Theorem 1.5 and so the routine iterate-PLM will produce approximations to each of 
the corresponding $d_{k} / 2$ roots of $f_{k}$ (with multiplicity). Such initial points $z_{j}$ will be produced by choose-4d-good-initial-points, as was shown in Section 2.2. As a consequence of Lemma 2.5, the good approximations iterate-PLM are approximate zeros of $\psi=f_{k}-\vec{\tau}$, with $\alpha_{\psi}<1 / 8$. As was discussed in Section 2.4, application of the routines select-approx-zeros and weed-out-duplicates will select exactly one representative for each approximated root of $\psi$, giving at least $d_{k} / 2$ such approximations $\lambda_{j}$. This selection is necessary since some of the initial $z_{j}$ which did not satisfy the hypothesis of Theorem 1.5 may still have converged.

As was shown in Section 2.6, the approximations produced yield an $\epsilon / 4^{d}$-factorization, since the each the $\lambda_{i}$ are made sufficiently close to the roots of $\psi$ by the routine polish-roots, and $\left\|\psi-f_{k}\right\|$ is sufficiently small. This completes the proof of Theorem 2.1.

\section{REFERENCES}

[Ahl79] L. Ahlfors, Complex Analysis, McGraw-Hill, 1979.

[BFKT88] M. Ben-Or, E. Feig, D. Kozen, And P. Tiwari, A fast parallel algorithm for determining all roots of a polynomial with real roots, SIAM J. Computing, (1988), pp. 10811092.

[BT90] M. BEN-Or AND P. TIWARI, Simple algorithms for approximating all roots of a polynomial with real roots, J. Complexity, (1990), pp. 417-442.

[BM75] A. Borodin And I. Munro, The Computational Complexity of Algebraic and Numeric Problems, American Elsevier Pub., 1975.

[DH69] B. Dejon and P. Henrici, eds., Constructive Aspects of the Fundamental Theorem of Algebra, Wiley Interscience, 1969.

[Fri90] J. Friedman, Random polynomials and approximate zeros of Newton's method, SIAM J. Computing, 19 (1990), pp. 1068-1099.

[Hen74] P. Henrici, Applied and Computational Complex Analysis, Wiley Interscience, 1974.

[JT70] M. A. Jenkins AND J. F. Traub, A three-stage variable-shift iteration for polynomial zeros and its relation to generalized Rayleigh iteration, Numer. Math., 14 (1970), pp. $252-263$.

[Kim88] M. KIM, On approximate zeros and rootfinding algorithms for a complex polynomial, Math. of Computation, 51 (1988), pp. 707-719.

[Kim89a] - Error analysis and bit complexity: Polynomial root finding problems I. Preprint, Bellcore, Morristown, NJ, 1989.

[Kim89b] _ Topological complexity of a root finding algorithm, J. Complexity, 5 (1989), pp. 331344.

[Man92] A. Manning, How to be sure of solving a complex polynomial using Newton's method, Boletim Soc. Bras. Mat., 22 (1992), pp. 157-177.

[Mar66] M. Marden, The Geometry of Polynomials, Amer. Math. Soc., 1966.

[Nef90] A. NEFF, Specified precision polynomial root isolation in in NC, in $31^{\text {st }}$ FOCS, 1990, pp. 152-162.

[Pan87] V. PAN, Sequential and parallel complexity of approximate evaluation of polynomial zeros, Comput. Math. Applic., 14 (1987), pp. 561-622.

[Ren87] J. RENEGAR, On the worst-case arithmetic complexity of approximating zeros of polynomials, J. Complexity, 3 (1987), pp. 90-113.

[Sch82] A. SchöNhAGE, The fundamental theorem of algebra in terms of computational complexity. Technical Report, Univ. of Tubingen, 1982.

[Shu83] M. SHUb, The geometry and topology of dynamical systems and algorithms for numerical problems. notes from lectures given at D.D.4 Peking University, Beijing, China, 1983.

[SS85] M. Shub And S. Smale, Computational complexity: On the geometry of polynomials 
and a theory of cost I, Ann. Sci. École Norm. Sup., 18 (1985), pp. 107-142.

[SS86] Computational complexity: On the geometry of polynomials and a theory of cost II, SIAM J. Comp., 15 (1986), pp. 145-161.

[SS93a] Complexity of Bezout's theorem I: Geometric aspects, J. Amer. Math. Soc, 6 (1993), pp. $459-501$.

[SS93b] Complexity of Bezout's theorem II: Volumes and probabilities, in Computational Algebraic Geometry, F. Eyssette and A. Galligo, eds., Progress in Mathematics, Vol. 109, Birkhauser, 1993, pp. 267-285.

[SS93c] - Complexity of Bezout's theorem III: Condition number and packing, J. Complexity, 9 (1993), pp. 4-14.

[SS93d] _ Complexity of Bezout's theorem IV: Probality of success, extensions. IBM research report, 1993.

[STW88] M. Shub, D. Tischler, And R. Williams, The Newtonian graph of a complex polynomial, SIAM J. Math. Anal, 19 (1988), pp. 246-256.

[Sma81] S. Smale, The fundamental theorem of algebra and complexity theory, Bull. Amer. Math. Soc., 4 (1981), pp. 1-36.

[Sma85] - On the efficiency of algorithms of analysis, Bull. Amer. Math. Soc., 13 (1985), pp. $87-121$.

[Sma86] _ Newton's method estimates from data at one point, in The Merging Disciplines: New Directions in Pure, Applied, and Computational Mathematics, Springer, 1986, pp. 185-196.

[Sut89] S. Sutherland, Finding Roots of Complex Polynomials with Newton's Method, PhD thesis, Boston University, 1989.

[Tis89] D. Tischler, Critical points and values of complex polynomials, J. Complexity, 5 (1989), pp. $438-456$. 